\title{
Multifocal Polypoid Endometriosis Mimicking Malignancy in a Young Woman with a History of Hormonal Treatment
}

\author{
Ji-Young Kim · Tae-Jong Song ${ }^{1} \cdot$ Hye-Kyung Choi ${ }^{2} \cdot$ Jeong Yun Shim \\ Department of Pathology, CHA Gangnam Medical Center, CHA University School of Medicine, Seoul; \\ ${ }^{1}$ Department of Obstetrics and Gynecology, Kangbuk Samsung Medical Center, Sungkyunkwan University School of Medicine, Seoul; \\ 2Department of Radiology, CHA Gangnam Medical Center, CHA University School of Medicine, Seoul, Korea
}

Polypoid endometriosis is a rare type of endometriosis that sometimes mimics the clinical presentation of malignancy. We report a case of a young woman with a history of hormone treatment who was suspected to have an ovarian malignancy with metastases.

\section{CASE REPORT}

A 30-year-old G0P0 woman with a history of left ovarian cystectomy due to endometriosis presented with a huge left adnexal mass detected on ultrasonography. After the previous cystectomy, she was administered a gonadotrophin releasing hormne $(\mathrm{GnRH})$ agonist for four months and then a combined oral contraceptive (OC), Mercilon (Organon Ltd., Doublin, Ireland) (mixed ethinylestradiol and desogestrel), for about two years. Her menstrual cycle was regular, but she experienced severe dysmenorrhea. The patient's serum cancer antigen (CA) 125 level was increased (595.3 U/mL), while her CA 19-9 and carcinoembryonic antigen results were normal.

Ultrasonography showed an 11-cm-sized, mixed-echoic, semisolid mass in the left adnexa. Abdominopelvic computed tomography revealed the mass to be solid with enhancement (Fig. 1A). Another mass was present in the left abdominal wall, extending to the peritoneum and the rectus abdominis muscle. A

\section{Corresponding Author}

Jeong Yun Shim, MD, PhD

Department of Pathology, CHA Gangnam Medical Center, 566 Nonhyeon-ro, Gangnam-gu, Seoul 06135, Korea

Tel: +82-2-3468-2612, Fax: +82-2-3468-2619, E-mail: jyshim@cha.ac.kr

Received: March 19, 2015 Revised: April 30, 2015

Accepted: May 12, 2015 small amount of ascites was observed in the perihepatic space (Fig. 1B).

A laparoscopic left salpingo-oophorectomy was performed to remove the 11-cm-sized tumor encasing and destroying the adnexal organs. Intraoperative frozen section examination indicated the lesion to be benign, most likely severe endometriosis. Other peritoneal foci were also resected.

Microscopically, the left adnexal mass was composed of endometrial-like tissue. Dilated endometrial-type glands were longitudinally arranged around grouped, thick-walled vessels, which were reminiscent of a typical endometrial polyp (Fig. 1C). Worrisome features, such as increased stromal cellularity or atypia, were not noted. The mitotic rate was less than 1 in 10 highpower fields (Fig. 1D). Immunohistochemically, both the epithelial and the stromal cells were positive for estrogen receptor and progesterone receptor. The stromal cells were also positive for CD10 (Fig. 1E, F). The absence of increased cellularity and/ or stromal atypia easily excluded the possibility of adenosarcoma. The endometrial-type stromal cells with CD10 positivity in our case were obviously different from the fibroblastic and CD34positive stromal cells in adenofibroma.

The patient did not present residual disease at her follow-up six months after the operation.

\section{DISCUSSION}

Polypoid endometriosis is a rare type of endometriosis. In contrast to the usual cystic endometriosis, it is solid and frequently forms a nodular polypoid mass that can sometimes mimic malignancy at presentation. ${ }^{1}$ Frequently involved sites are the vagi- 

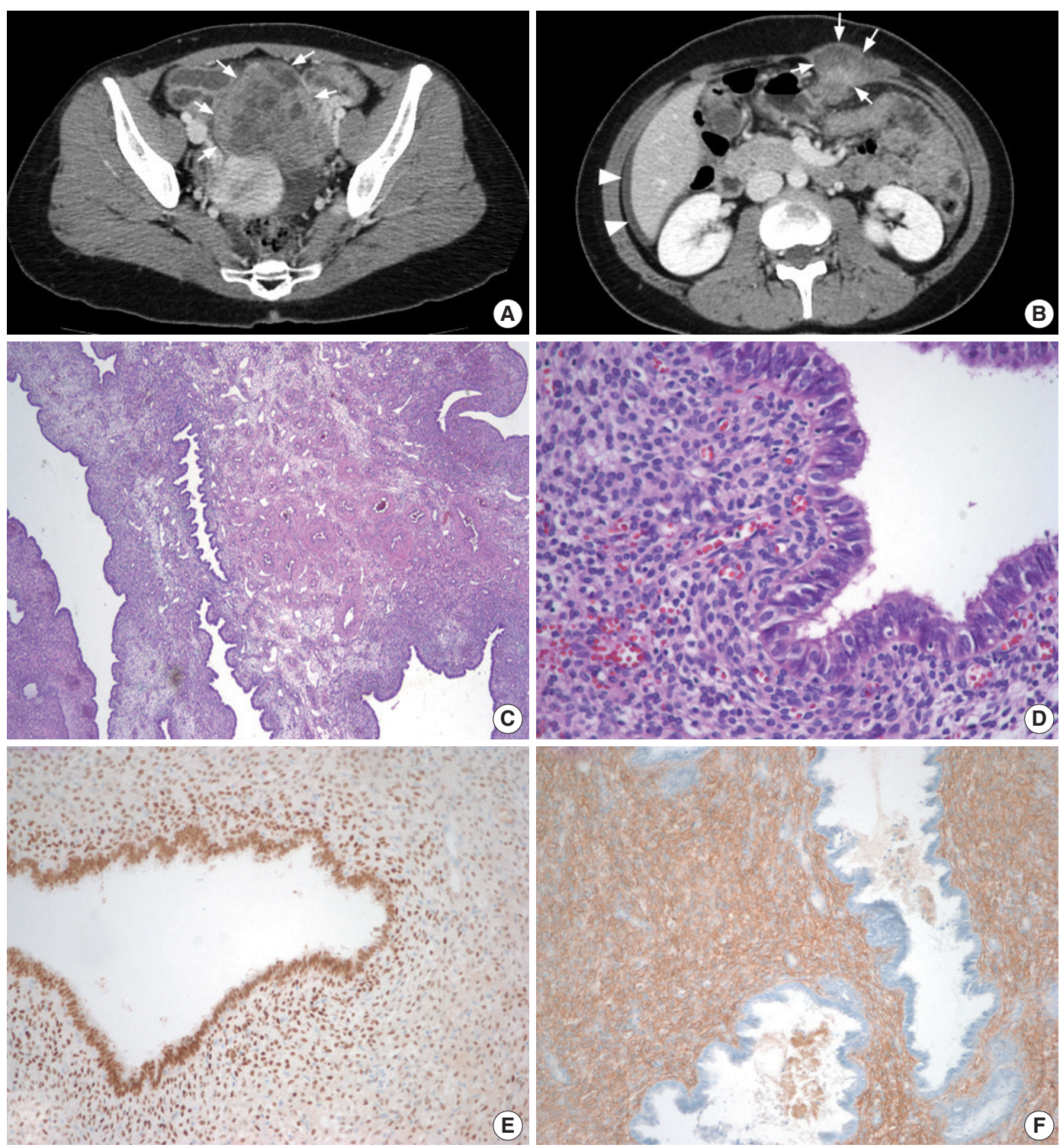

Fig. 1. (A) On axial computed tomography, a solid and cystic mass (arrows) with periuterine adhesions is visible in the left adnexa. (B) Another mass can be seen in the left upper abdominal wall (arrows) and extending to the rectus abdominis muscle. A small amount of ascites is present in the perihepatic area (arrowheads). (C) Microscopically, the mass is composed of endometrial-like tissue. Dilated endometrial-type glands are longitudinally arranged around grouped, thick-walled vessels with swollen and congested stroma, which is reminiscent of a typical endometrial polyp. (D) Some glandular epithelial cells demonstrate ciliated metaplasia. (E) On immunohistochemical staining, the glandular epithelial cells and some stromal cells are positive for estrogen receptor. (F) CD10 is positive only in stromal cells.

na, cervix, adnexa, and colorectum. ${ }^{2-4}$ It usually causes non-specific symptoms, such as dysmenorrhea, menorrhagia, or vaginal spotting. ${ }^{1,3,5}$

The etiology of polypoid endometriosis is uncertain. Some reported cases have been associated with unopposed estrogen treatment, phytoestrogen, and tamoxifen, suggesting the role of estrogenic stimulation in the development of the disease. ${ }^{1,3-6}$ Withdrawal of a GnRH agonist was also reported to cause polypoid endometriosis as a rebound phenomenon. ${ }^{3,7}$ To our knowledge, there is no previous report of polypoid endometriosis associated with OCs. In a collective review of 22 cases of polypoid endometriosis, Parker $e t$ al. ${ }^{1}$ included four cases with mixed estrogenprogestin therapy. But it was in the form of hormone replacement therapy after menopause or oophorectomy rather than a contraceptive use. OCs, which are frequently used to prevent the recurrence of endometriosis after conservative surgery, have also been reported to increase the risk of endometriosis in certain conditions. ${ }^{8}$ In our patient, estrogen seems to have retained its 
stimulatory effect despite being balanced with progesterone.

Polypoid endometriosis can sometimes mimic the clinical presentation of malignancy. ${ }^{1}$ The presence of a large adnexal mass, multiple peritoneal and pelvic foci, ascites, and elevated CA 125 in our case gave the preoperative impression of a malignant ovarian tumor with peritoneal metastases. An elevated CA 125 level is one of the laboratory abnormalities associated with endometriosis and is also reported in some cases of polypoid endometriosis. ${ }^{4,9}$ It has been reported that ascites, though very rarely, can be associated with endometriosis, especially in the presence of severe disease. ${ }^{10}$ When it presents with ascites, particularly accompanied by multiple peritoneal lesions on radiology, adnexal polypoid endometriosis can be easily misinterpreted as ovarian malignancy with peritoneal metastases.

Our patient underwent laparoscopic surgery despite the preoperative suspicion of malignancy. Gynecological laparoscopy is increasingly performed, even in some early oncological conditions, because of its reduced invasiveness. In our case, an intraoperative frozen diagnosis of a benign mass prevented conversion of the procedure to a more extensive surgery with laparotomy. Despite the relatively typical microscopic findings, the confusing presentation of the mass, such as its large size, solid nodular nature, destruction of normal anatomical structures, multifocality, peritoneal involvement, ascites, and elevated CA 125, rendered doubt on the benign nature of the lesion. Pathologists' awareness of this rare form of endometriosis is vital in order to avoid unnecessary radical surgery. Yet again, a thorough microscopic examination with sufficient sampling is warranted in these patients in order to exclude complex hyperplasia or adenocarcinoma, which, although very rare, has arisen in polypoid endometriosis. ${ }^{1}$ Polypoid endometriosis should also be included in the differential diagnosis of seemingly malignant adnexal tumors in a young woman, especially when associated with a history of hormone therapy, notably OCs, for endometriosis.

\section{Conflicts of Interest}

No potential conflict of interest relevant to this article was reported.

\section{REFERENCES}

1. Parker RL, Dadmanesh F, Young RH, Clement PB. Polypoid endometriosis: a clinicopathologic analysis of 24 cases and a review of the literature. Am J Surg Pathol 2004; 28: 285-97.

2. Jaiman S, Gundabattula SR, Pochiraju M, Sangireddy JR. Polypoid endometriosis of the cervix: a case report and review of the literature. Arch Gynecol Obstet 2014; 289: 915-20.

3. Othman NH, Othman MS, Ismail AN, Mohammad NZ, Ismail Z. Multiple polypoid endometriosis: a rare complication following withdrawal of gonadotrophin releasing hormone (GnRH) agonist for severe endometriosis: a case report. Aust N Z J Obstet Gynaecol 1996; 36: 216-8.

4. Kraft JK, Hughes T. Polypoid endometriosis and other benign gynaecological complications associated with tamoxifen therapy: a case to illustrate features on magnetic resonance imaging. Clin Radiol 2006; 61: 198-201.

5. Felix A, Nogales FF, Arias-Stella J. Polypoid endometriosis of the uterine cervix with Arias-Stella reaction in a patient taking phytoestrogens. Int J Gynecol Pathol 2010; 29: 185-8.

6. Chang CK, Chen P, Leu FJ, Lou SM. Florid polypoid endometriosis exacerbated by tamoxifen therapy in breast cancer. Obstet Gynecol 2003; 102(5 Pt 2): 1127-30.

7. Marugami N, Hirohashi S, Kitano S, et al. Polypoid endometriosis of the ureter mimicking fibroepithelial polyps. Radiat Med 2008; 26: $42-5$.

8. Tu FF, Du H, Goldstein GP, Beaumont JL, Zhou Y, Brown WJ. The influence of prior oral contraceptive use on risk of endometriosis is conditional on parity. Fertil Steril 2014; 101: 1697-704.

9. Laird LA, Hoffman JS, Omrani A. Multifocal polypoid endometriosis presenting as huge pelvic masses causing deep vein thrombosis. Arch Pathol Lab Med 2004; 128: 561-4.

10. Gungor T, Kanat-Pektas M, Ozat M, Zayifoglu Karaca M. A systematic review: endometriosis presenting with ascites. Arch Gynecol Obstet 2011; 283: 513-8. 